\title{
Neuromorphic Walking Gait Control
}

\author{
Susanne Still \\ University of Hawaii at Manoa, Information and Computer Sciences, 1680 \\ East-West Road, Honolulu, HI 96822, USA \\ Klaus Hepp, Rodney J. Douglas \\ Institute for Neuroinformatics, ETH Zürich/University of Zürich, Winterthurerstr. \\ 190, CH-8057 Zürich, Switzerland
}

\begin{abstract}
We present a neuromorphic pattern generator for controlling the walking gaits of four-legged robots which is inspired by central pattern generators found in the nervous system and which is implemented as a VLSI chip. The chip contains oscillator circuits that mimic the output of motor neurons in a strongly simplified way. We show that four coupled oscillators can produce rhythmic patterns with phase relationships that are appropriate to generate all four-legged animal walking gaits. These phase relationships together with frequency and duty cycle of the oscillators determine the walking behavior of a robot driven by the chip, and they depend on a small set of stationary bias voltages. We give analytic expressions for these dependencies. This chip reduces the complex, dynamic inter-leg control problem associated with walking gait generation to the problem of setting a few stationary parameters. It provides a compact and low power solution for walking gait control in robots.
\end{abstract}

\section{Index Terms}

Walking gait control, four-legged walking gaits, neuromorphic engineering, Central Pattern Generators, autonomous walking robot.

\section{INTRODUCTION}

Legged robots, like animals, should be able to make use of different walking gaits in appropriate response to terrain, speed and task. In general, this should help to simplify movement tasks, e.g.

To whom correspondence should be addressed at sstill@ hawaii.edu 
turning, and also to lower energy consumption at a given speed - in analogy to what has been observed in horses [1]. The coordination of movements required for successful locomotion is a complex problem involving many degrees of freedom. Walking gait control can be split into two control problems, that of the individual leg movement and that of inter-leg control. The latter determines the phase relationship between the legs and hence the walking gait, in accordance with the definition of gait established by Muybridge [2] and later refined by Alexander [3]. The idea underlying the work we present here is to simplify the inter-leg control problem by splitting it into two stages: (i) A pattern generator that transforms a small set of stationary parameters into rhythmic outputs that can be used to drive the motors of a legged robot to yield a rhythmic walking pattern (i.e gait) and (ii) a learning machine which enables the robot to learn those parameters that lead to a desired gait. We have demonstrated the utility of this system in an earlier, short paper [4] and here we elaborate in detail on the pattern generator.

It was realized early in the development of autonomous walking machines that computing the inverse kinematics to control a legged machine is extremely CPU intensive [5] and that distributed motor control schemes are more efficient [6]-[8]. Those ideas were largely inspired by biological findings on motor control, an area that was for most of the 20th century dominated by the attempt to decide between two controversial hypotheses stating that rhythmic movements, such as walking, are controlled either by reflex chains or by central pattern generators (CPGs). CPGs are centers in the nervous system that create rhythmic output even if they are not connected to any sensors. Many groups have found those pattern generators in different animals and showed - for many behaviors including swimming, walking and breathing - that neural activity was sustained in isolation experiments (for a review see [9]). These findings, together with some theoretical understanding of how networks of coupled oscillators generate patterns with different symmetries that can be mapped to different walking gaits (see, for example, [10]-[13]), have inspired many walking controllers for robot locomotion (for example [14]-[19]) as well as some neuromorphic central pattern generators, e.g. [20]-[26].

The approach we take here is different from previous CPG inspired walking controllers in two ways. Whereas most CPG inspired controllers that have been used for robot locomotion are simulations of model neurons run on a general purpose computer [14]-[18], our pattern generator 


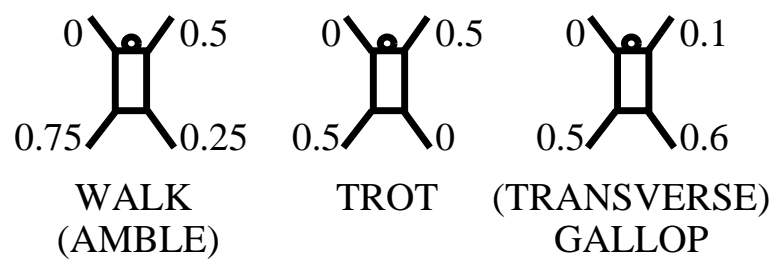

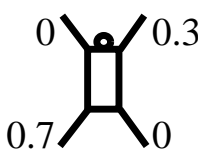

CANTER

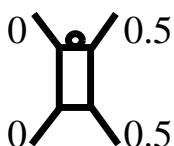

PACE

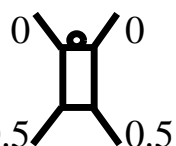

BOUND

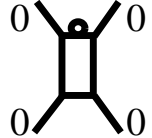

PRONK

Fig. 1. Gaits used by four-legged walking animals, distinguished with respect to the relative phase relationships (in units of $2 \pi$ ) between their legs. The front left leg serves as a reference point. Redrawn from [3].

is a neuromorphic system, compact and small, implemented as VLSI (very large scale integrated) circuits on a chip. Compact solutions like this with low power consumption are important for the future of autonomous robots. In contrast to other neuromorphic pattern generators, where the CPG was build from silicon neurons implemented with different levels of detail [20]-[26], we make our pattern generator as simple as possible. On one hand, this reduces the number of control parameters. On the other hand, the particular beauty of our approach is that the circuits in the oscillators of the CPG are so simple that they can be described by linear differential equations which can be solved analytically and therefore we understand the functional relationship between the control voltages and the output of the CPG which in turn we know how to map onto the walking behavior of a machine.

The oscillator circuits we use mimic motor neurons in a strongly simplified way. They are inspired by and very similar to circuits which were first used for robotic locomotion by Tilden (see e.g. [27] and for an analysis of those circuits [28]). The output waveforms of the oscillator network are abstractions of neuronal bursts, capturing in square wave functions only those values of the neuronal output which are relevant for controlling the inter-leg coordination in a robot. These are (i) the burst duration, (ii) the inter burst frequency and (iii) the phase lag between the onsets of bursts of different neurons. The corresponding quantities that characterize the output waveforms of the oscillator networks are (i) the duty cycle of each oscillator, (ii) the 
oscillator frequency, and (iii) the phase lags between oscillators. ${ }^{1}$ The circuits on our chip, being significantly more compact than previous neuromorphic CPG neurons, are nevertheless sufficient to control inter-leg coordination, and furthermore, their square wave output voltages can directly control dc-motors, such that no additional motor-neuron layer is necessary. Instead of allowing functional reconfigurations of the CPG, as is done in [26] to produce different walking gaits, we ask which fixed architecture contains the fewest oscillators and can still produce all observed four-legged animal gaits. We find such a minimal architecture for a pattern generator in Sec. II-C. Switching between gaits is simply implemented by changing control parameters.

We use Alexander's classification of gaits in which a walking gait is defined solely by the phase lags between the rhythmic movements of the legs. Figure 1 illustrates the quadrupedal walking gaits distinguished by Alexander. Walk, trot and gallop are gaits used by many animals and transitions between them often occur as the animal increases its speed [1]. The pace gait is used by camels and the bound gait by animals like the Siberian souslik [29]. Although, in this paper, we focus on four-legged locomotion, it is easy to see that the pattern generator could be extended to more than four legs. We have chosen quadruped locomotion because four-legged animals have walking patterns, like the canter gait, which are less symmetric and hence more difficult to generate than those of, for example, six-legged animals.

\section{Neuromorphic Walking Gait Control Chip}

The Gait Control (GC) chip takes a set of stationary voltages as input parameters and produces for each value in parameter space a rhythmic set of output voltages with certain phase relationships, duty cycles and a certain common frequency. The output voltages are step functions that can be used to drive dc-motors which move the joints of a robot. In our case, to control walking gaits, they move the hip joints of a robot. Therefore, the phase relationships between the output voltages determine the walking gait of the robot. The common frequency sets the step frequency and the duty cycles of the motors can be used to control the walking direction of the robot: if all duty cycles are identical, then any symmetric robot will walk forward. However, robots with certain mechanical properties will turn in response to an asymmetry in the duty cycles of leg movements. In the following circuit analysis we will compute as a function of the input parameters: (i) the

\footnotetext{
${ }^{1}$ In the entire paper, we give phase lags in units of $2 \pi$.
} 


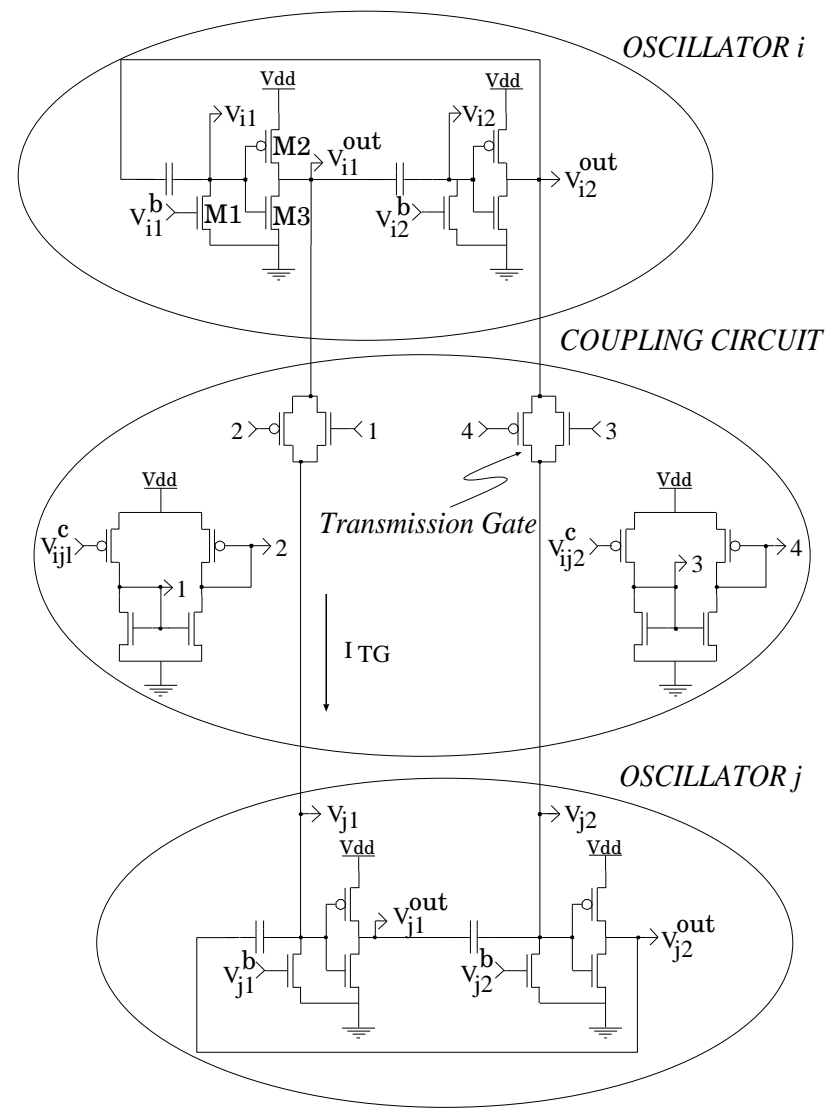

Fig. 2. Circuit diagram for the oscillators and coupling circuits on the GC chip. Each oscillator consists of two identical subcircuits and has two bias voltages, $V_{i 1}^{b}$ and $V_{i 2}^{b}$, which control frequency and duty cycle. Two oscillators, $i$ and $j$, are coupled with a transmission gate. This coupling affects only oscillator $j$. The gate voltage on the $n$-fet of each transmission gate is set to be the complementary voltage of the p-fet of the same transmission gate with the circuits drawn next to the transmission gates. The two control voltages $V_{i j 1}^{c}$ and $V_{i j 2}^{c}$ are copied to nodes 2 and 4, respectively, while the voltages at nodes 1 and 3 are $\left(V_{d d}-V_{i j 1}^{c}\right)$ and $\left(V_{d d}-V_{i j 2}^{c}\right)$, respectively. On the chip are two pairs of coupled oscillators, with inputs $V_{i 1}$ and $V_{i 2}$, and outputs $V_{i 1}^{\text {out }}$ and $V_{j 1}^{\text {out }}$. The chip furthermore contains one uncoupled oscillator with output $V_{i 1}^{\text {out }}$ and two additional coupling circuits with inputs $V_{i 1}^{\text {out }}$ and $V_{i 2}^{\text {out }}$ and outputs $V_{j 1}$ and $V_{j 2}$ (not shown here, see Fig. 3). All input- and output lines and bias voltages are connected to pads.

frequency and duty cycle of an uncoupled oscillator (Sec. II-A) and (ii) the phase lag between two coupled oscillators (Sec. II-B). We will analyze the behavior of pattern generators with a chain-of-oscillators architecture (Sec. II-C) and compare it to a ring-of-oscillators architecture (Sec. II-D). Our circuit analysis predicts that a chain of $n$ coupled oscillators suffices to control the walking gaits of an $n$ legged machine. Experimental results verify this for $n=4$ (Sec. III). 

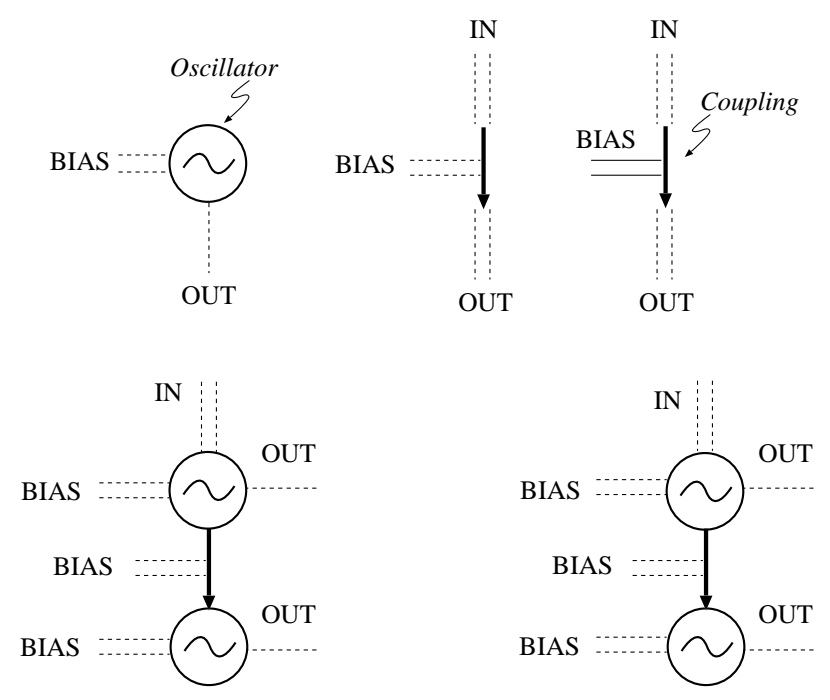

Fig. 3. Sketch of the components on the chip. The chip contains two pairs of coupled oscillators. The circuit for them is shown in Fig. 2. Round symbols correspond to the oscillator circuits and arrows to the coupling circuits in Fig. 2. Additionally, there are two free coupling circuits and one uncoupled oscillator. All dotted lines are connected to pads. A chain of four oscillators is implemented by connecting the output of the slave oscillator of one of the pairs to one input line of a free coupling circuit and the inverted voltage output to the other input line. The output of the coupling circuit is connected to the input of the master oscillator of the other pair of coupled oscillators. A ring of four oscillators is implemented by connecting also the output of the second pair to the input of the first pair in the same way via the other free coupling circuit.

The GC chip consists of oscillator circuits and circuits that couple two oscillators. At frequencies useful for locomotion, the coupling is uni-directional, affecting only one of the oscillators. On the chip, there are two pairs of coupled oscillators (with two input lines and two output lines), one single oscillator that is not connected to any other circuit on chip (with one output line), and two unconnected coupling circuits (with two input lines and two output lines). Input- and output lines are connected to pads and hence the components on the chip can be connected off-chip in different ways. That allows for the implementation and study of controllers with different architectures. A circuit diagram for two coupled oscillators is given in Fig. 2, and a sketch of all components on the chip is shown in Fig. 3. The uncoupled oscillator is identical to the oscillator circuit in the top row of Fig. 2, while the additional coupling circuits on the chip follow the same circuit diagram as given in the middle row of Fig. 2. All oscillators on the chip have only one output line, because the other output voltage is always the mirror image. It is therefore unnecessary to connect it to another pad, instead we can use an inverter off-chip to reproduce 
the second output. The layout is extremely compact, containing less than 80 transistors, leaving room for possible extensions to larger networks of oscillators on one chip. On this chip, however, we have a large empty area which we used for on-chip capacitors. The size of these capacitors is not a limiting factor because, as we will see in the following, as long as the capacitors are all identical, the phase lags between oscillators do not depend on the value of the capacitance (see (26)). ${ }^{2}$ Since the oscillation frequency of the networks we propose to use for gait control depends on the time constant in the circuit, smaller capacitors could be compensated by adjusting the bias voltages of the transistors in the circuit to smaller voltages. ${ }^{3}$ This would, within a large voltage range, not violate the assumptions we are making in the circuit analysis and hence our results would still hold. Alternatively, off-chip capacitors could be used. The chip was fabricated using a CMOS $1.2 \mu$ process.

\section{A. The Oscillator circuit}

The oscillator segregates into two identical, reciprocally connected sub-circuits. Each sub-circuit consists of an inverter (transistors M2 and M3 in the upper left part of Fig. 2), a capacitor, C, and an $\mathrm{n}$-fet transistor, M1, connected between the input node of the inverter and ground. The gate voltages $V_{i 1}^{b}$ and $V_{i 2}^{b}$ in Fig. 2 are the control parameters of the oscillator. An oscillation is triggered by a step from ground to the supply voltage at either one of the two output nodes ${ }^{4}$, $V_{i k}^{o u t}$. Such a step occurs when the chip is powered up, making the oscillator circuit self-triggered. The oscillation can be stoped by opening the two transistors that are connected to ground.

To compute how frequency and duty cycle of the oscillator depend on the bias voltages of the transistors we focus on one sub-circuit of the oscillator. Transistor M1 operates in the subthreshold regime, since the gate voltage, denoted by $V_{k}^{b}$, has to be smaller than $0.8 \mathrm{~V}$ to produce an output waveform in the frequency range that is needed to generate locomotion (approx. 0.5

\footnotetext{
${ }^{2}$ Neither do the duty cycles depend on the capacitance (see(9) and (25)), and therefore the control over these circuit outputs would not be more difficult if smaller capacitors were used.

${ }^{3}$ Control of the oscillation period would not be harder with smaller capacitors (this point is further elaborated in the footnote following equation (7)).

${ }^{4}$ The subscript $k \in\{1,2\}$ labels here and in the following the two sub-circuits of an oscillator. Later, the subscripts $i$ and $j$ label different oscillators in a network. For now we can drop the index $i$ since we are only considering one oscillator.
} 


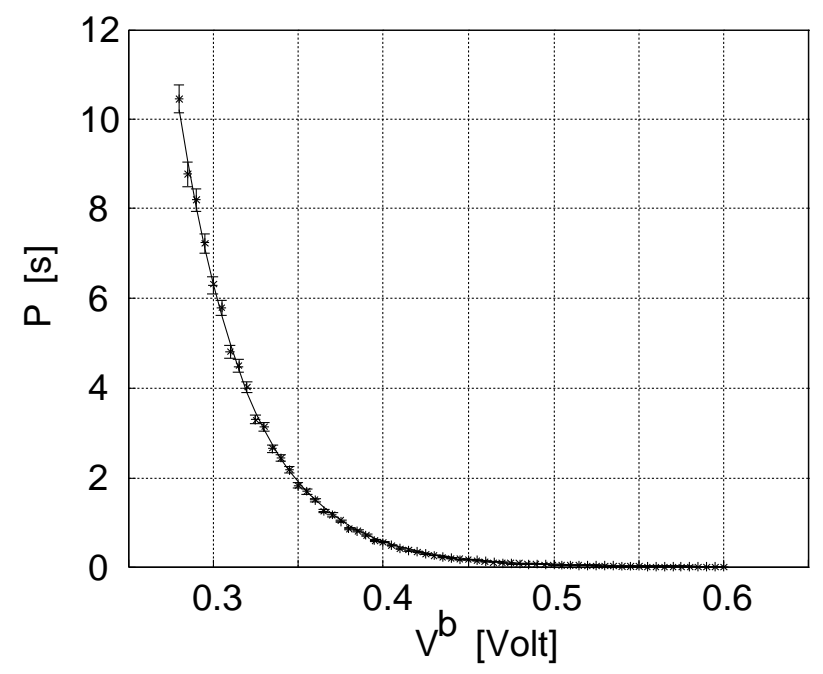

Fig. 4. Period of an (uncoupled) oscillator as a function of the bias voltage. For simplicity the two bias voltages of the oscillator are set to the same value, $V^{b}:=V_{1}^{b}=V_{2}^{b}$, which means that the duty cycle of the oscillation is $50 \%$. The oscillation period follows (7) (solid line). All the capacitors on the chip have a capacitance of $C=515.99 \times 10^{-12} F$, according to the process parameters. The values of $V_{\max }, I_{0 n}$ and $\kappa$ are estimated with a least squares fit: $V_{\max }=3.23 \mathrm{~V}, I_{0 n}=2.21 \times 10^{-16} \mathrm{~A}$ and $\kappa=0.6202$.

$\mathrm{Hz}$ to $2 \mathrm{~Hz}$ ). Let $t=0$ be the time after voltage $V_{\bar{k}}^{\text {out }}$ has risen from ground to $V_{d d}$, the supply voltage. The subscript $\bar{k}$ denotes the side of the oscillator not equal to $k$, i.e $\bar{k}=2$ if $k=1$ and vice versa. The transient rise at $V_{\bar{k}}^{\text {out }}$ causes $V_{k}$ to rise to the voltage $V_{k}(t=0)=V_{\max }$. Note that we neglect here and in the following treatment any transient behavior at the on- and off-sets and assume that both on- and off-sets take no time. We can do this, because the time constants with which the inverters switch are much smaller than the period of the oscillation suitable for a robotic locomotion application.

We define the signal time $T_{k}$ as the time it takes the voltage $V_{k}$ at the input node of the inverter to decay from its maximum value $V_{\max }$ at time $t=0$ to the threshold voltage of the inverter, $V_{t h}$, i.e. $V_{k}\left(t=T_{k}\right)=V_{t h}$. Then, by definition, $V_{k} \geq V_{t h}$ during the time $0 \leq t \leq T_{k}$. All p- and $\mathrm{n}$-fet transistors on the GC chip have the same widths and the same lengths and hence $V_{t h}$ is given by [30]

$$
V_{t h}=\frac{V_{d d}+V_{t p}+V_{t n} \sqrt{\mu_{n} / \mu_{p}}}{1+\sqrt{\mu_{n} / \mu_{p}}}=1.345 \mathrm{~V}
$$


where $V_{t p}$ and $V_{t n}$ are the threshold voltages for the inverter's p-fet and n-fet transistors, respectively. $\mu_{p}$ and $\mu_{n}$ denote the mobility of the charge carriers. From $V_{t h}>4 k_{B} T / q$ it follows that $V_{k}>4 k_{B} T / q$ for $0 \leq t \leq T_{k}$, where $T$ is the temperature, $k_{B}$ the Boltzmann constant and $q$ the charge. Transistor M1 therefore operates in saturation and the drain current through M1 is given by [31].

$$
I=I_{0} \exp \left(\gamma V_{k}^{b}\right)
$$

with

$$
\gamma:=\frac{q}{k_{B} T} \kappa
$$

where $\kappa$ is the subthreshold slope coefficient and $I_{0}$ is the zero bias current. During the time $0 \leq t \leq T_{k}$, the voltage at node $V_{\bar{k}}^{\text {out }}$ is constant and equal to $V_{d d}$, and Kirchhoff's current law implies for the voltage $V_{k}(t)$

$$
\dot{V}_{k}(t)=-\frac{I_{0}}{C} \exp \left(\gamma V_{k}^{b}\right)
$$

where $C$ denotes the capacitance. Note that all capacitors on the chip are identical. We solve (4) with the initial condition $V_{k}(t=0)=V_{\text {max }}$, substitute $V_{k}\left(t=T_{k}\right)=V_{t h}$, solve the resulting equation for $T_{k}$ and obtain

$$
T_{k}=\left(V_{\max }-V_{t h}\right) \frac{C}{I_{0}} \exp \left(-\gamma V_{k}^{b}\right)
$$

The period of the oscillator is the sum of both half-cycles

$$
P=T_{1}+T_{2}=\frac{C}{I_{0}}\left(V_{\text {max }}-V_{t h}\right)\left[\exp \left(-\gamma V_{1}^{b}\right)+\exp \left(-\gamma V_{2}^{b}\right)\right]
$$

and the oscillation frequency is given by $\nu=1 / P$. In (6), we have assumed that $V_{\text {max }}$ and $V_{t h}$ are the same for both sub-circuits. This is a reasonable approximation, given that the layout of both sub-circuits is identical.

For the case that $T_{1}=T_{2}$, the duty cycle of the oscillation is $50 \%$. This is implemented by setting the bias voltages on both sides of the oscillator to the same value $V_{1}^{b}=V_{2}^{b}=: V^{b}$. Assuming that all components are the same on both sides of the oscillator, the period becomes 
(compare Fig. 4) 5

$$
P_{\text {sym }}=\frac{2 C}{I_{0}}\left(V_{\text {max }}-V_{t h}\right) \exp \left(-\gamma V^{b}\right){ }^{6}
$$

In general, we define the duty cycle with respect to $T_{k}$, as

$$
D_{k}=T_{k} / P
$$

which depends on the difference $V_{k}^{b}-V_{\bar{k}}^{b}$ of the bias voltages on both sides of the oscillator circuit:

$$
D_{k}=\left(1+\exp \left(\gamma\left(V_{k}^{b}-V_{\bar{k}}^{b}\right)\right)\right)^{-1}
$$

\section{B. The Coupling circuit}

Two oscillators are coupled by two transmission gates, one on each side of the oscillator circuit, connected between the output node of one oscillator and the input node to the inverter of the other oscillator (see Fig. 2). At the low frequencies which are relevant for locomotion, the coupling only affects the second oscillator, because the inverters of the first oscillator act as impedance buffers. For ease of reference let us call the first oscillator the master oscillator and denote its parameters and voltages with subscripts $i$, while we call the second oscillator the slave oscillator, labeled with subscripts $j$. The bias voltages of the p-fet transistors of the transmission gates, denoted by $V_{i j k}^{c}$, control the coupling strength.

1) Phase lag between two oscillators: The resistive coupling induces a time delay $T^{D}$ between the edges of the square wave oscillations in both oscillators. In general, the oscillator circuits (including the coupling circuits) do not have to have identical values of bias voltages on both sides. Therefore, the duty cycle of each oscillator can differ from $50 \%$ and there are two delays times, $T_{k}^{D}$ and $T_{\bar{k}}^{D}$ (see Fig. 5). For ease of reference, we thus define two phase lags, one for each half of the oscillation

$$
\phi_{j k}=\frac{T_{k}^{D}+T_{j k}}{P}=\frac{T_{k}^{D}}{P}+D_{j k}
$$

\footnotetext{
${ }^{5}$ All measurements in this paper are taken at roughly room temperature. We did not carefully control the temperature beyond the usual temperature regulation of the building.

${ }^{6}$ With smaller capacitors, the control over the period would not be more difficult - the exponential decay is controlled by the coefficient $\gamma$. The range of obtainable frequencies however does change with $C$ : $P_{\text {high }}=P\left(V^{b}=0\right)=\frac{2 C}{I_{0}}\left(V_{\text {max }}-V_{t h}\right)$. But, periods of up to ten seconds (which is certainly slow enough for most walking applications) would still be obtained if the capacitors were roughly six orders of magnitude smaller than the ones on this chip (using the numerical values in the caption of Fig. 3).
} 


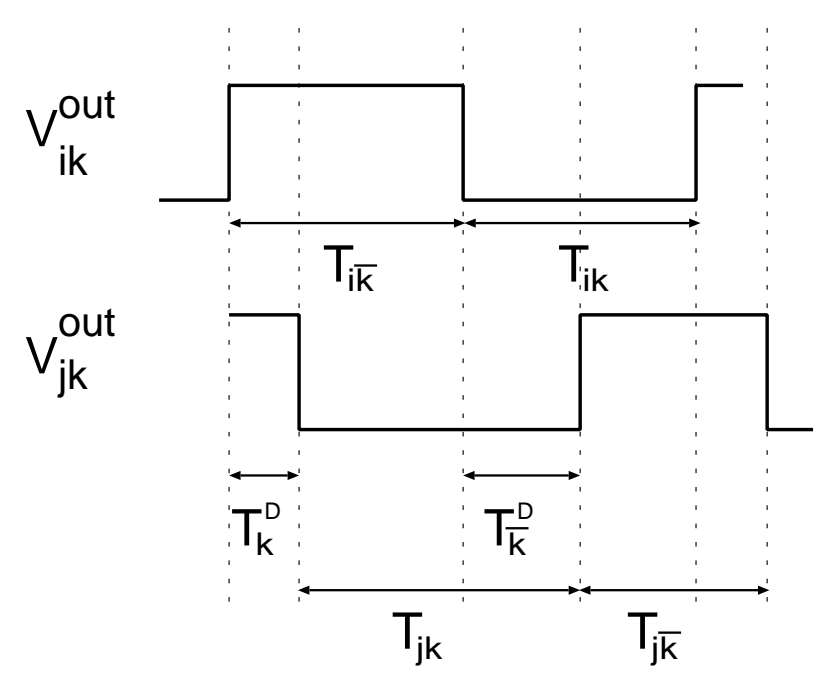

Fig. 5. Sketch of the output traces of two coupled oscillators. Signal times and delay times are indicated.

where $D_{j k}=T_{j k} / P$ is duty cycle of oscillator $j$ and $P$ is the common oscillation period of both oscillators. Having a common period is important for generating walking gaits. For all values of the bias voltages that are shown in Fig. 6, the oscillators have a common period.

To compute the delay times $T_{k}^{D}$ as a function of the bias voltages of the transistors, we make the approximation that all transistors operate in saturation. For this, we have to assume that $V_{j k}(t=0) \simeq 0.1 \mathrm{~V}$. This is a good approximation of the true initial value, if there is enough time for the voltage $V_{j k}$ to discharge, between the last time $V_{j \bar{k}}^{o u t}$ switched from $V_{d d}$ to ground and the time $t=0$. This can be assumed to be the case for the locomotion frequency range. We now consider the following situation: a switch from low to high occurs in the output voltage of oscillator $i, V_{i k}^{\text {out }}$, while the voltage $V_{j k}$ is small. $V_{j k}$ is the voltage at the node in oscillator $j$ that oscillator $i$ couples into. This situation is characterized by

$$
\begin{aligned}
& V_{i k}^{\text {out }}=\left\{\begin{aligned}
0 & \text { for } \quad t<0 \\
V_{d d} & \text { for } \quad 0 \leq t \leq T_{k}^{D}
\end{aligned}\right. \\
& V_{j \bar{k}}^{\text {out }}=0 \text { for } 0 \leq t \leq T_{k}^{D} \\
& V_{j k}(t=0)=V_{0} \simeq 0.1 \mathrm{~V}
\end{aligned}
$$

With these assumptions, the current through the transmission gate is given by

$$
I_{T G}=I_{0 n} \exp \left(\gamma\left(V_{d d}-V_{i j k}^{c}\right)\right) \exp \left(-\frac{q}{k T} V_{j k}\right)+I_{0 p} \exp \left(\gamma\left(V_{d d}-V_{i j k}^{c}\right)\right)
$$


The current through the single transistor (M1) is the same as before (compare (2)) and Kirchhoff's current law implies

$$
\dot{V}_{j k}(t)=A \exp \left(-\frac{q}{k T} V_{j k}(t)\right)+B
$$

with

$$
\begin{array}{r}
A=\frac{I_{0 n}}{C} \exp \left(\gamma\left(V_{d d}-V_{i j k}^{c}\right)\right) \\
B=\left[I_{0 p} \exp \left(\gamma\left(V_{d d}-V_{i j k}^{c}\right)\right)-I_{0 n} \exp \left(\gamma V_{j k}^{b}\right)\right] / C
\end{array}
$$

We solve (15) with the initial condition $V_{j k}(t=0)=V_{0}$, substitute $V_{j k}\left(T_{k}^{D}\right)=V_{t h}$ and solve for $T_{k}^{D}$. We obtain

$$
T_{k}^{D}=\frac{\alpha}{\mu \exp \left(-\gamma V_{i j k}^{c}\right)-\exp \left(\gamma V_{j k}^{b}\right)} \ln \left[\frac{\rho\left(V_{t h}\right) \exp \left(-\gamma V_{i j k}^{c}\right)-\sigma\left(V_{t h}\right) \exp \left(\gamma V_{j k}^{b}\right)}{\rho\left(V_{0}\right) \exp \left(-\gamma V_{i j k}^{c}\right)-\sigma\left(V_{0}\right) \exp \left(\gamma V_{j k}^{b}\right)}\right]
$$

with

$$
\begin{aligned}
\alpha & =\frac{k_{B} T}{q} \frac{C}{I_{0 n}} \\
\mu & =\frac{I_{0 p}}{I_{0 n}} \exp \left(\gamma V_{d d}\right) \\
\rho(V) & =\left[I_{0 n}+I_{0 p} \exp \left(\frac{q}{k T} V\right)\right] \exp \left(\gamma V_{d d}\right) \\
\sigma(V) & =I_{0 n} \exp \left(\frac{q}{k T} V\right)
\end{aligned}
$$

From (18) we can see that $T_{k}^{D}$ is only defined if the argument of the logarithm in (18) is larger than zero. This yields a constraint for the sum $V_{j k}^{b}+V_{i j k}^{c}$

$$
\begin{aligned}
& V_{i j k}^{c}+V_{j k}^{b}<\frac{k_{B} T}{q \kappa} \ln \left[\left(\exp \left(-\frac{q}{k T} V_{t h}\right)+\frac{I_{0 p}}{I_{0 n}}\right) \exp \left(\gamma V_{d d}\right)\right] \\
& \text { or } \\
& V_{i j k}^{c}+V_{j k}^{b}>\frac{k_{B} T}{q \kappa} \ln \left[\left(\exp \left(-\frac{q}{k T} V_{0}\right)+\frac{I_{0 p}}{I_{0 n}}\right) \exp \left(\gamma V_{d d}\right)\right]
\end{aligned}
$$

Rough numerical values for the bounds are

$$
V_{i j k}^{c}+V_{j k}^{b}<4.75 \quad \text { or } \quad V_{i j k}^{c}+V_{j k}^{b}>4.86
$$

Thus, there is a small region of approximately $100 \mathrm{mV}$ in which $T_{k}^{D}$ diverges. We have observed this singularity experimentally, however, it occurs outside of the range of bias voltages applied for the use of the chip in the context of locomotion (compare Fig. 6). 

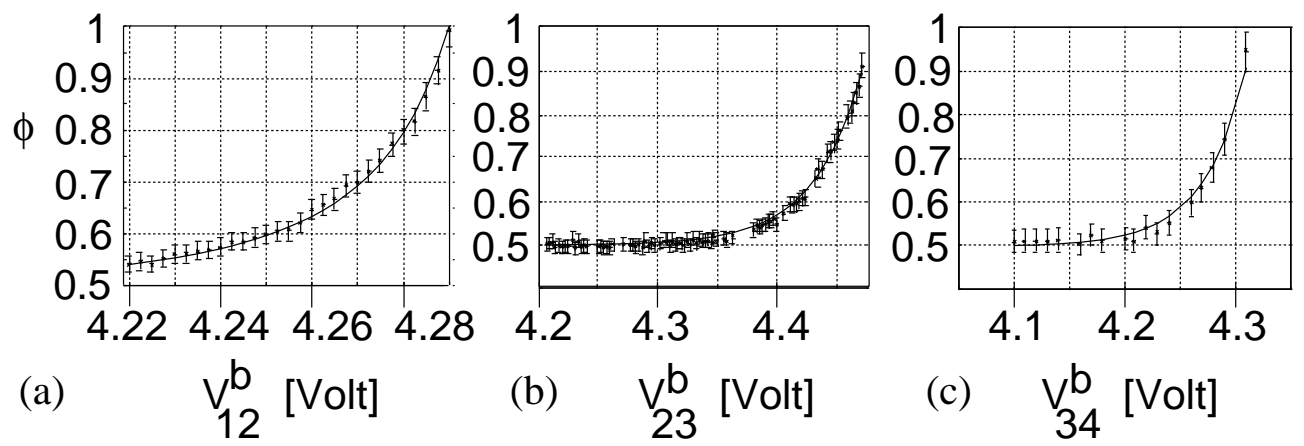

Fig. 6. Phase lags (in units of $2 \pi$ ) between (a) the first two oscillators, (b) the second and third oscillator, and (c) the third and forth oscillator in a chain of 4 oscillators. They follow (26) (solid lines). In (a), the parameters $V_{\max }$ and $I_{0 n}$ for the first oscillator are those ones determined in Fig. 4 . $I_{0 p}$ is estimated by a least squares fit: $I_{0 p}=1.56 \times 10^{-19}$ A. (b) $I_{0 p}=6.50 \times 10^{-18}$ A and $I_{0 n}=1.0 \times 10^{-17} \mathrm{~A}$. (c) $I_{0 p}=1.4 \times 10^{-19} \mathrm{~A}$ and $I_{0 n}=2.0 \times 10^{-17} \mathrm{~A}$.

\begin{tabular}{|l||c|c|c|c|c|c|c|}
\hline Gait & walk & trot & canter & gallop & pace & bound & pronk \\
\hline$\phi_{1}$ & 0.75 & 0.5 & 0.7 & 0.5 & 0 & 0.5 & 0 \\
\hline$\phi_{2}$ & 0.75 & 0 & 0.6 & 0.6 & 0.5 & 0.5 & 0 \\
\hline$\phi_{3}$ & 0.75 & 0.5 & 0.7 & 0.5 & 0 & 0.5 & 0 \\
\hline
\end{tabular}

TABLE I

PHASE LAGS NECESSARY TO PRODUCE WALKING GAITS IF A CHAIN OF FOUR OSCILLATORS IS CONNECTED TO THE MOTORS THAT DRIVE THE LEGS OF A ROBOT AS DESCRIBED IN THE TEXT.

$T_{k}^{D}$ is zero when the transmission gate opens $\left(V_{i j k}^{c} \rightarrow 0\right)$. Then, the term in front of the logarithm in (18) becomes $\alpha /\left(\mu-\exp \left(\gamma V_{j k}^{b}\right)\right)$. The denominator of this term is dominated by $\mu$, which is on the order of $10^{56}$. Thus this term vanishes and therefore $T_{k}^{D} \rightarrow 0$.

\section{Chains of coupled oscillators}

An additional oscillator can be connected to the two coupled oscillators of Sec. II-B such that the slave of Sec. II-B is the master to the third oscillator. And we can continue in this fashion to create chains of $n$ oscillators. For each of them, the results from Sec. II-B apply, i.e. the common period of the system is set by the master oscillation, given by (6), the duty cycle of which is given by (9), and the phase lags in the system are given by (10) together with (9) and 
(18). The duty cycle of the $(i+1)$-th oscillator in the chain is set by the duty cycle of the $i$-th oscillator, and by the delay times $T_{j k}^{D}$ between oscillators $i$ and $j=(i+1)$, via

$$
D_{j k}=D_{i \bar{k}}+\frac{1}{P}\left(T_{j \bar{k}}^{D}-T_{j k}^{D}\right) .
$$

If we consider a symmetric circuit with duty cycles of $50 \%$ then the functional dependence of the phase lags between oscillators on the parameters simplifies and it is instructive to write the equations out. ${ }^{7}$ Equation (10) becomes $\phi_{j}=T^{D} / P+1 / 2$ and substitution of (7) and (18) yields

$$
\phi_{j}=\frac{1}{2}\left(\frac{\lambda \exp \left(\gamma\left(V_{1}^{b}-V_{j}^{b}\right)\right)}{\mu \exp \left(-\gamma\left(V_{i j}^{c}+V_{j}^{b}\right)\right)-1} \ln \left[\frac{\rho\left(V_{t h}\right)-\sigma\left(V_{t h}\right) \exp \left(\gamma\left(V_{i j}^{c}+V_{j}^{b}\right)\right)}{\rho\left(V_{0}\right)-\sigma\left(V_{0}\right) \exp \left(\gamma\left(V_{i j}^{c}+V_{j}^{b}\right)\right)}\right]+1\right)
$$

With $\lambda=k_{B} T /\left(q\left(V_{\max }-V_{t h}\right)\right)$. If the bias voltages for all oscillators are the same $\left(V_{j}^{b}=\right.$ $\left.V_{1}^{b} \forall j \in\{2, . ., n\}\right)$, then $\phi_{j}$ depends only on the sum of $V_{1}^{b}$ and the bias voltage of the transmission gate $V_{i j}^{c}$. The factor before the logarithm reduces to $\lambda /\left(\mu \exp \left(-\gamma\left(V_{i j}^{c}+V_{1}^{b}\right)\right)-1\right) \approx$ $\lambda / \mu \exp \left(\gamma\left(V_{i j}^{c}+V_{1}^{b}\right)\right)$ and the dominating dependence on $V_{i j}^{c}+V_{1}^{b}$ is exponential (compare Fig. $6)$.

The phase shift between two oscillators ranges between 0.5 and 1 as can be seen from Fig. 6. The lower bound arises because the delay time is lower bound by zero, while the upper bound is enforced by the constraint that all oscillations should have the same frequency. We have seen period doubling at higher values of the coupling voltages, and this could be useful for very specific applications, for example as in [25], where the knee flexor moves at double the frequency as the knee extensor and the hip. However, in this article, we are primarily interested in walking gait control and therefore limit our analysis to movements with a common oscillation frequency. The GC chip's step function outputs are used to move dc-motors back and forth, and thus the range of phase shifts is equivalent with and easily transformed to the range 0 to 0.5 by switching the polarity of a motor. This way the range can also be extended to the full range at the cost of one extra bit of information per motor. We can drive the legs of a walking machine with a chain of four coupled oscillators that is arranged in the following way: the master oscillator drives the front left leg, the second oscillator in the chain drives the hind left leg, the

\footnotetext{
${ }^{7}$ By a symmetric circuit we mean one in which all parameters with $k=1$ have the same values as those with $k=2$; we can thus drop the index $k$.
} 
(a)

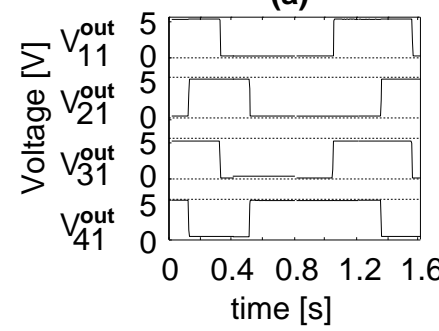

(b)

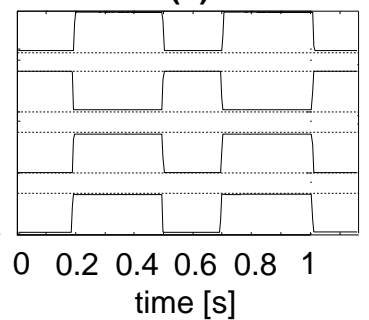

Fig. 7. Output waveforms of a ring of four oscillators. (a) mode 1; (b) mode 2. The bias voltages of all oscillators are set to $0.4 \mathrm{~V}$, and all coupling voltages are at $4.3 \mathrm{~V}$.

third drives the front right leg and the forth the hind right leg. Then, the challenge of producing the quadrupedal walking gaits, as shown in Fig. 1, reduces to producing the phase lags shown in Table I. We see immediately from the curves in Fig. 6 that the pattern generator will produce any of the gaits walk, canter, trot, gallop, pace, bound and pronk - given the correct control voltages. A complete set of all gaits is given in Sec. III together with measurements of the leg movements of a robot that is controlled by this oscillator network.

\section{Rings of coupled oscillators}

Rings of coupled oscillators are often used as a model to explain the generation of animal walking gaits [11], [32]. The main idea is that the change in one global parameter, like the common frequency or the coupling strength, leads the system through a series of different gaits via symmetry breaking bifurcations. The oscillators are usually assumed to be nonlinear and by far more complex than the ones we have implemented here. In our case, a ring of $n$ oscillators is formed from a chain by coupling the output of the $n$-th oscillator into the first oscillator which was the master oscillator of the chain. Since now each oscillator is enslaved by one other oscillator, the common oscillation frequency is no longer given by equation (6). At frequencies interesting for robotic locomotion we find bi-stability for most combinations of the control parameters. Two different modes occur, one of which (mode 1) can be mapped to a walk gait (Fig. 7(a)). The second mode (mode 2) occurs with a higher frequency (Fig. 7(b)) and can be mapped to a fast trot gait. To use both gaits in one system, we need to switch polarity on some of the motors.

Analysis of related circuits [28] suggests that to achieve more complex behavior and more 


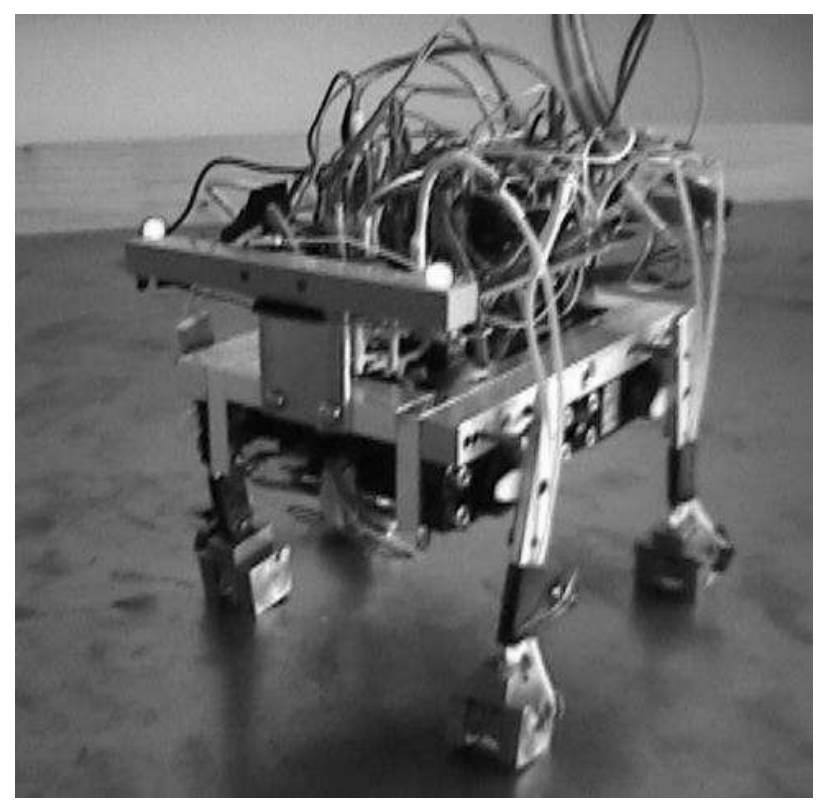

Fig. 8. Image of the walking machine

gaits, one needs to use rings of larger size. Altogether, the performance of a ring controller made from these types of oscillators is rather limited compared to the range of gaits that a chain of 4 oscillators can generate and the straightforward control we have over them.

\section{LEARNING TO WALK}

The CPG controller we have introduced here can produce patterns for controlling all of the observed animal gaits. We map the voltage output patterns of the chip to the movements of a robot by using them directly to drive dc-motors at the hip joints of a four legged robot. The output of each oscillator therefore corresponds directly to moving the respective leg back and forth. Stance phase is implemented by magnetic feet, which turn on during the backward movement and turn off during the swing phase, thus breaking the symmetry and propelling the robot in one direction. We use this extremely simple machine (Fig. 8), because it allows us, with a kinematic model, to analytically calculate the trajectory of the robot, given the output of the chip. Together with the analytical results in Sec. II, this gives us an analytical understanding of how the control parameters of the chip map to the movement of the robot. It is out of the scope of the present paper to present the kinematic model, we refer the interested reader to [28]. However, it is important to point out qualitatively, how the output parameters of the chip control 

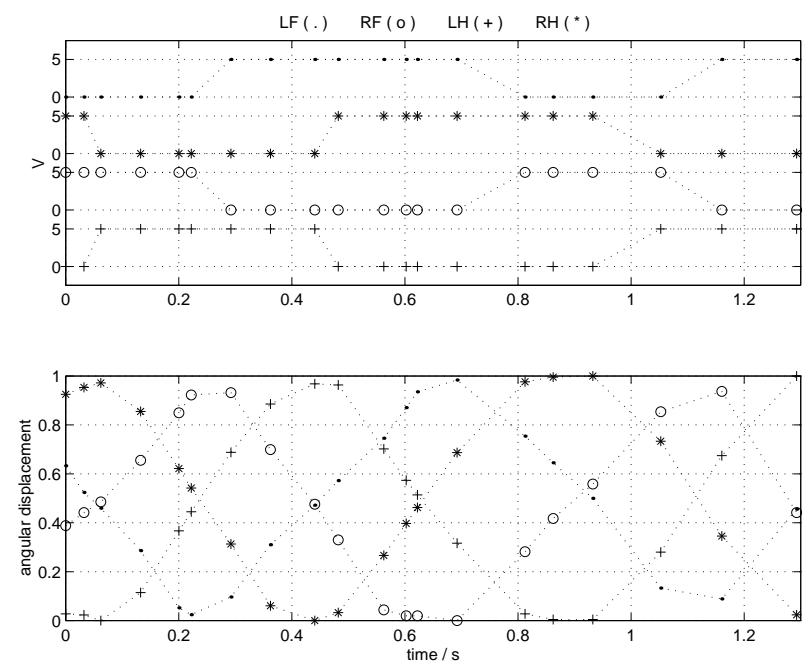

Fig. 9. Walk, $P=0.89$ s. The legs move with a phase shift of 0.25 in succession: LF, RH, RF, and finally LH.
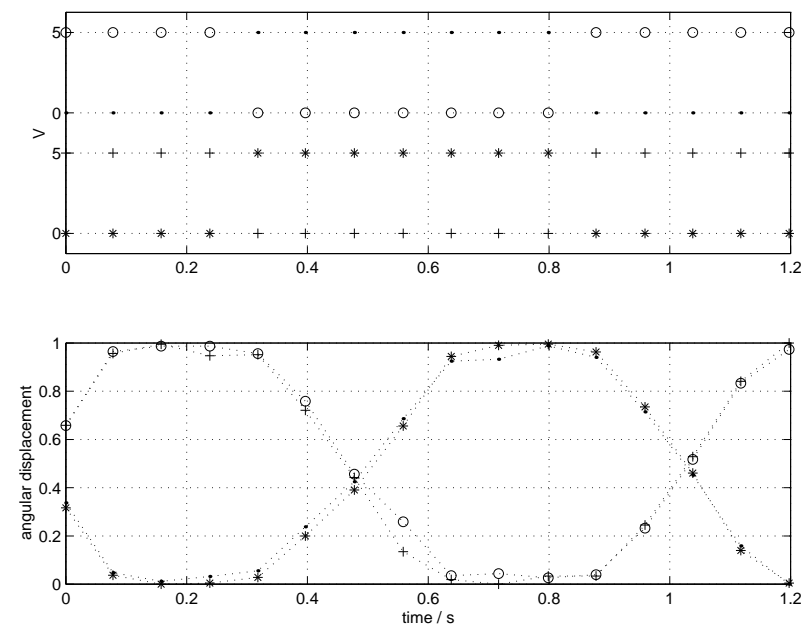

Fig. 10. Trot, $P=1.1$ s. Legs LF and RH move in phase with each other, legs RF and LH move in phase with each other and legs LF and RF move out of phase.

the walking pattern of the robot: The common frequency of the oscillators corresponds to the step frequency. The duty cycle of an oscillator corresponds to the fraction of the time in a cycle during which the leg is in stance versus swing phase. Identical duty cycles thus result in forward locomotion, while breaking the symmetry between the duty cycles of the legs on the right and 

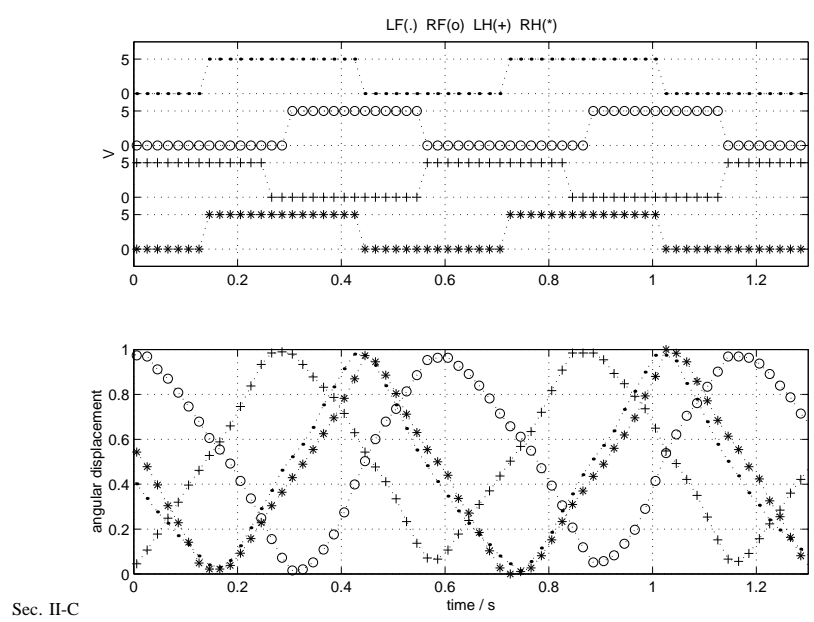

Fig. 11. Canter. $P=0.58$ s. Legs LF and RH move in synchrony. Leg RF follows with a phase shift of 0.3 (with respect to LF) and LH follows with a phase shift of 0.7 (with respect to LF).
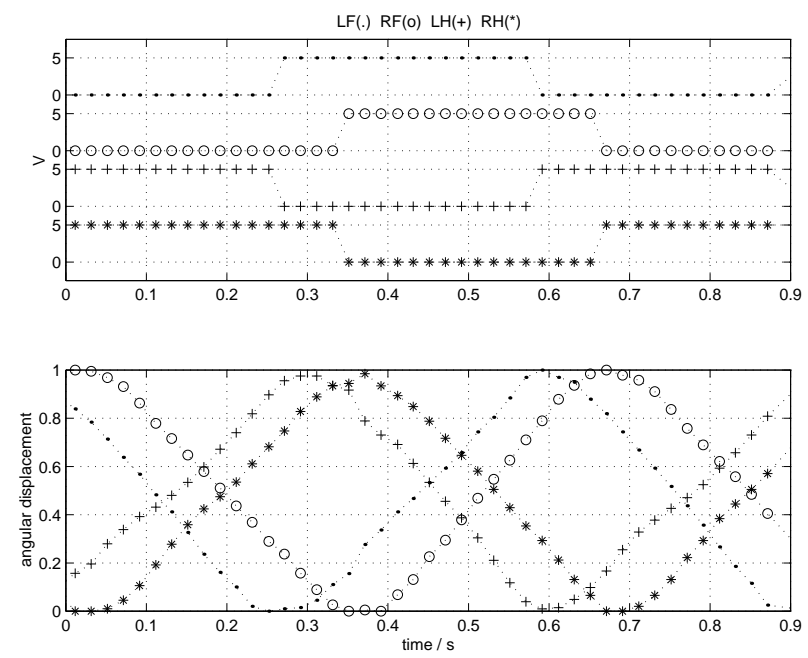

Fig. 12. Transverse Gallop. $P=0.63 \mathrm{~s}$. The legs move in succession LF, RF, LH, RH with phase lags of 0.1 between LF and $\mathrm{RF}$ as well as between $\mathrm{LH}$ and $\mathrm{RH}$, and 0.5 between LF and LH.

on the left side of the robot results in turning. ${ }^{8}$ The phase lags between the oscillators determine the gait as discussed in Sec. II-C and listed in Table I.

Although we have calculated explicit analytical relationships between the control parameters of the chip and its output parameters (and thus the gait of the robot), due to transistor mismatch

${ }^{8}$ For experimental results on turning we refer to [28]. 

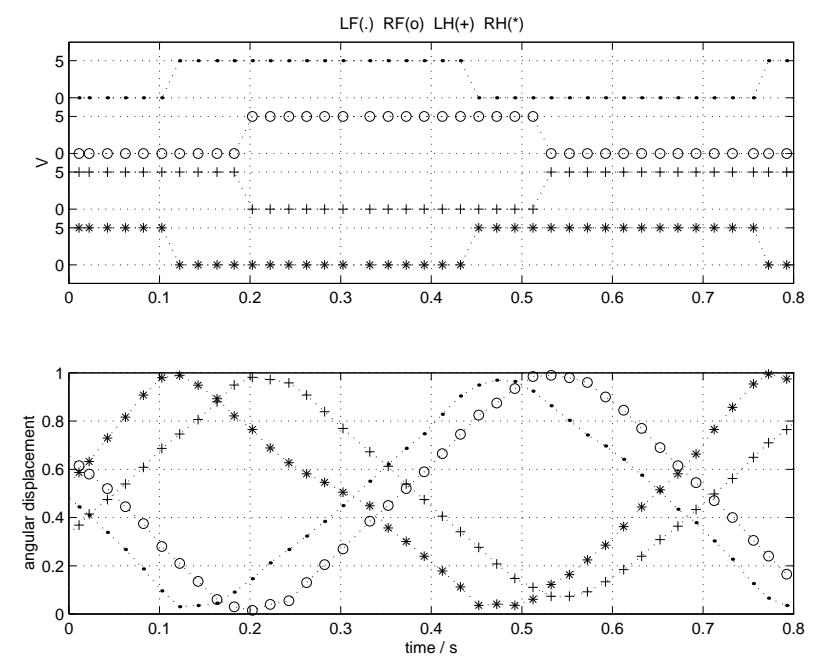

Fig. 13. Rotary Gallop. $P=0.65$ s. The legs move in succession LF, RF, RH, LH with phase lags of 0.1 between LF and RF as well as between RH and LH, and 0.5 between LF and RH.

and related problems [31], [33], these values can only be used to set a range in which to search for the desired output pattern, and the parameters have to be fine tuned. Instead of fine tuning by hand, we use a learning procedure which is based on an unsupervised Support Vector Machine algorithm [34] to learn the parameter regime in which the desired gait occurs and we then set the bias voltages close to the center of gravity of this region to maximize gait stability. The region in parameter space is large enough to ensure that the gait is stable, extending typically over a few millivolts to a few tens of millivolts across each dimension. This learning procedure allows the robot to learn gaits automatically and autonomously (i.e. without requiring fine tuning by the experimenter), and it is an elegant way of automatically fine tuning parameters to deal with the problem of transistor mismatch. In principle, this idea could be carried over to many aVLSI systems which require fine tuning of many parameters, such as spiking neurons [35], [36]. For details on the learning procedure, we refer to [4], [28].

Results of the walking patterns are shown in Figs. 9 to 16. The four traces in the upper panels are the outputs of the four oscillators and the four traces in the lower panels reflect the position of the respective legs. We measure the voltage at potentiometers which are attached to each motor at each hip joint and this voltage (which is normalized to the interval $[0,1]$ ) is proportional to the angular displacement of the leg. An increase in angle means that the leg moves backward with respect to the body's direction of movement, thus propelling the body 

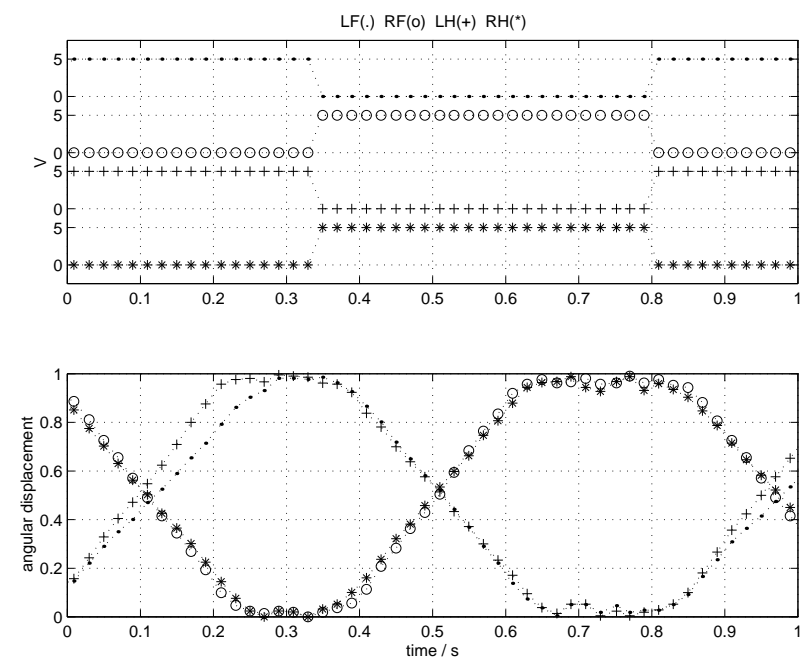

Fig. 14. Pace. $P=0.85 \mathrm{~s}$. Leg pairs on the same side of the body move in synchrony. There is a phase shift of 0.5 between the two leg pairs.

forward (stance phase). The four legs are distinguished by the following abbreviations in the caption and symbols in the plot: left front leg (LF; dots), right front leg (RF; circles), left hind leg (LH; crosses) and right hind leg (RH; stars). The four traces of the leg movements are overlaid to aid the discrimination of the phase relationships between them. Raw data is plotted, which is subject to noise caused by both the mechanics of the setup, in particular the potentiometers, and by the data acquisition, which does not sample at a high enough frequency. ${ }^{9}$ The numbers given in the captions for the period, $P$, and the phase lags are obtained by averaging over 20 periods. Thus the data displayed can deviate slightly from these numbers. The phase relationships of the four voltage traces obtained from the potentiometers characterize the gait of the robot. For completeness, Figs. 12 and 13 show results for transverse and rotary gallop, respectively. We omitted this distinction in the introduction for simplicity, but there are two gallops, one in which the legs move as in Fig. 1, this is the transverse gallop. In the rotary gallop, the legs move in the succession LF, RF, RH, LH and the phase lags are 0.1 between LF and RF, 0.5 between LF and RH, 0.6 between LF and LH. Altogether, the data in Figs. 9 to 16 confirms that each of the animal gaits generated by the output of the GC chip (as discussed in Sec. II-C) is actually expressed in the movement of the robot.

\footnotetext{
${ }^{9}$ For details on the custom made data acquisition system we refer to [28].
} 

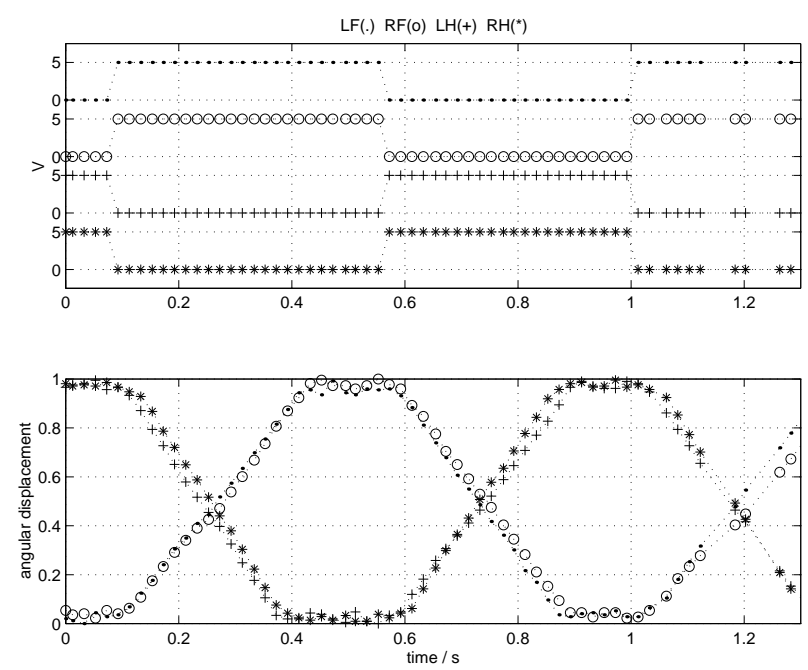

Fig. 15. Bound. $P=0.93 \mathrm{~s}$. Both legs of the same girdle move in synchrony. There is a phase shift of 0.5 between the two leg pairs.

To produce these results, we did not have to learn the ten bias voltages that we can individually control in a chain of four oscillators. Instead, we set all bias voltages $V_{j k}^{b}$ on one side of the oscillator (with the same value of the subscript $k$ ) to the same value: $V_{j k}^{b}=V_{k}^{b} ; \forall j=1, . ., 4$. There are now two parameters, $V_{1}^{b}$ and $V_{2}^{b}$, which control the common frequency of the network and the duty cycle of the master oscillator. ${ }^{10}$ The remaining six control voltages on the couping circuits are responsible for the duty cycles of the remaining three oscillators and for the three phase lags in the chain. We therefore control eight variables individually (common period, four duty cycles, three phase lags) with eight parameters.

\section{Discussion}

Networks made from oscillators of the kind discussed in this paper can be used to control the walking patterns of a four-legged robot by driving the motors that actuate the hips of the robot or alternatively by triggering whatever rhythmic motion the legs execute. A chain of four oscillators is the smallest circuit that allows for the generation of all quadrupedal walking gaits. The chip we presented here serves to reduce the complex, dynamic coordination problem involved in

\footnotetext{
${ }^{10}$ In the data shown here, this duty cycle is $50 \%$, causing the robot to walk straight forward. This allows further elimination of one parameter, but in general, we have to keep both parameters.
} 

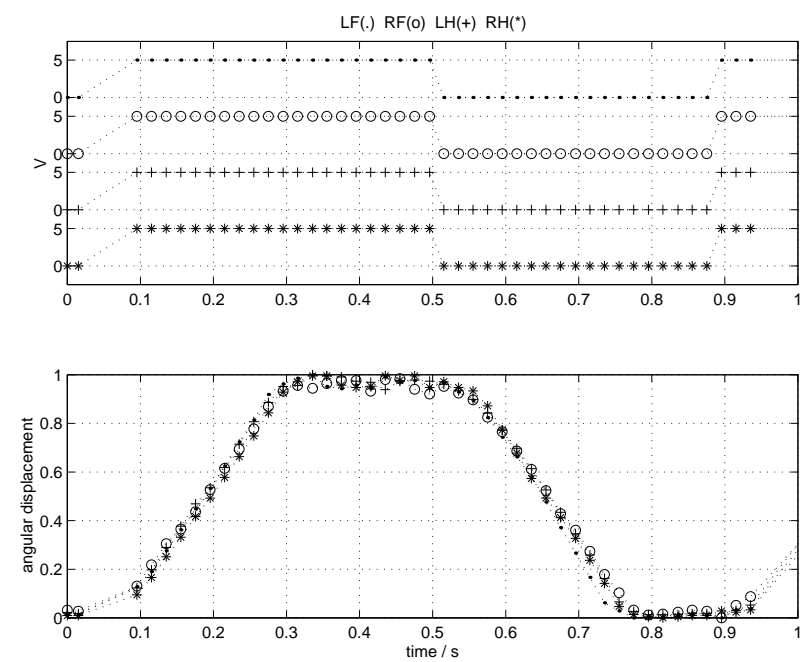

Fig. 16. Pronk. $P=0.86$ s. All four legs move in synchrony.

generating the walking gaits to the determination of a small set of control parameters which are applied as stationary voltages. We gave analytic expressions for the frequency, duty cycle and phase lag as a function of those control voltages.

Due to transistor mismatch, fine tuning of the control voltages is required. We solve this problem using a learning procedure that gives the robot full autonomy.

Extension to six-legged machines is straightforward, using a chain of 6 instead of 4 oscillators for inter-leg control. Walking gaits that require phase shifts smaller than 0.5 can easily be realized by switching the polarity on the output of an oscillator at the cost of one extra bit of information per oscillator. Extension to multi-jointed robots is also possible. Since the coupling is unidirectional, we can attach to every oscillator of the original chain another chain of oscillators. For example, for a four-legged robot with three joints per limb, we would drive the hip joints with a chain of four oscillators and within each leg the knee would be driven by an oscillator that is enslaved by the oscillator that drives the hip. The ankle in turn would be driven by an oscillator enslaved by the one that drives the knee. However, one has to be careful about the particular movement that is intended. For all rhythmic movements with the same common frequency, the extension of our circuits is straightforward, but if movements require one or several oscillators in the network to move at a different period then the others, more work would be needed to explore period doubling effects at strong coupling. 
As we have seen, the phase lags are smooth functions of the respective control parameters, and therefore transitions between different gaits can either be smooth or abrupt, implemented by a slow or a fast change of the control parameters, respectively. Having this flexibility can be of great advantage in real world applications as abrupt transitions are useful for fast gait changes while slow transitions might be more appropriate for example when the robot is turning while it is making the gait transition.

Sensory feedback can be incorporated into the controller simply via a change in the control parameters of the chip, because those parameters directly determine the walking behavior, as we have shown. Since the control parameters are voltages, they can be changed by sensory feedback provided by any device, the output of which, either is or can be transformed into an analog voltage. Those devices include photo diodes, potentiometers and other simple sensors as well as more complex, neuromorphic sensors like silicon retinas. Future research could explore the usage of the GC chip in combination with a variety of such sensory devices.

The GC chip does not require the robot to have specific mechanical characteristics. It can be used on a large spectrum of walking machines because its output characteristics can be adapted and learned by learning the control voltages. This makes the pattern generator we have presented here broadly useful.

\section{ACKNOWLEDGMENTS}

We wish to thank Adrian Whatley for helping with programming the data aquisition system that was used to aquire the data shown in Sec. III, and for many helpful discussions and comments on an earlier draft of this paper. For countless helpful discussions and comments, we are also indebted to Giacomo Indiveri, Bernhard Schölkopf, Shi-Chii Liu, Tobi Delbrück, the late Misha Mahowald and the late Jörg Kramer. Important inspiration and help has come from Mark W. Tilden and Gert Cauwenberghs. For instructive discussions we furthermore thank Anthony Lewis, Andreas Andreou, and Benjamin Arthur. 


\section{REFERENCES}

[1] D. F. Hoyt and R. C. Taylor, "Gait and the energetics of locomotion in horses," Nature, vol. 292, pp. 239-240, 1981.

[2] E. Muybridge, Animals in Motion. Dover Publications, Inc., New York, 1957.

[3] R. M. Alexander, Locomotion of Animals. Blackie, Glasgow and London, 1982.

[4] S. Still, B. Schölkopf, K. Hepp, and R. J. Douglas, "Four-legged Walking Gait Control Using a Neuromorphic Chip Interfaced to a Support Vector Learning Algorithm," in Advances in Neural Information Processing Systems 13, T. K. Leen, T. G. Dietterich, and V. Tresp, Eds. MIT Press, 2001, pp. 741-747.

[5] R. B. McGhee and G. Iswandi, "Adaptive Locomotion of a Multilegged Robot over Rough Terrain," IEEE Trans. on Systems, Man and Cybernetics, vol. 9, pp. 176-182, 1979.

[6] M. Donner, Real Time Control of Walking. Birkauser, Boston, 1987.

[7] R. Brooks, “A Robot that Walks; Emergent Behaviours from a Carefully Evolved Network," Neural Computation, vol. 1:2, pp. 365-382, 1989.

[8] H. Cruse, T. Kindermann, M. Schumm, J. Dean, and J. Schmitz, "Walknet - a biologically inspired network to control six-legged walking," Neural Networks, vol. 11, pp. 1435-1447, 1998.

[9] F. Delcomyn, "Neural Basis of Rhythmic Behavior in Animals," Science, vol. 210, pp. 492-498, 1980.

[10] M. Golubitsky, I. Stewart, P. L. Buono, and J. J. Collins, “A modular network for legged locomotion,” Physica D, vol. 115, pp. 56-72, 1998.

[11] J. J. Collins and I. N. Stewart, "Coupled Nonlinear Oscillators and the Symmetries of Animal Gaits," J. Nonlin. Sci., vol. 5, pp. 349-392, 1993.

[12] J. J. Collins and S. A. Richmond, "Hard-wired central pattern generators for quadrupedal locomotion," Biol. Cybern., vol. 71, pp. 375-385, 1994.

[13] G. Schöner, W. Y. Jiang, and J. A. S. Kelso, “A Synergetic Theory of Quadrupedal Gaits and Gait Transitions,” J. Theor. Biol., vol. 142, pp. 359-391, 1990.

[14] R. D. Beer, H. Chiel, R. Quinn, and K. Espenschied, "Mechanisms in stick insects applied to hexapod robot locomotion," in IEEE Conference on Robotics and Automation, 1992.

[15] R. D. Beer and H. Chiel, "Simulations of Cockroach Locomotion and Escape," in Biological Neural Networks in Invertebrate Neuroethology and Robotics. Academic Press, Inc., Boston, 1993.

[16] F. Pfeiffer, J. Eltze, and H.-J. Weidemann, "Six-legged technical walking considering biological principles," Robotics and Autonomous Systems, vol. 14, pp. 223-232, 1995.

[17] A. Fujii, N. Saito, K. Nakahira, and A. Ishiguro, "Generation of an Adaptive Controller CPG for a Quadruped Robot with Neuromodulation Mechanism," in Proceedings of the 2002 IEEE/RSJ Intl. Conference on Robots and Systems, EPFL, Lausanne, Switzerland, 2002.

[18] Y. Fukuoka, T. Mimura, N. Yasuda, and H. Kimura, "Integration of Multi Sensors for Adaptive Walking of a Quadruped Robot," in IEEE Conference on Multisensor Fusion and Integration for Intelligent Systems, 2003.

[19] R. Reeve and J. Hallam, “An Analysis of Neural Models for Walking Control,” IEEE Transactions on Neural Networks, vol. 16, no. 3, 2005.

[20] S. Ryckebusch, J. M. Bower, and C. A. Mead, "Modelling small oscillating biological networks in analog VLSI," in Advances in Neural Information Processing Systems 1. MIT Press, 1989, pp. 384-393.

[21] S. Ryckebusch, M. Wehr, and G. Laurent, "Distinct Rhythmic Locomotor Patterns can be generated by a Simple Adaptive Neural Circuit: Biology, Simulation, and VLSI Implementation,” J. Comp. Neurosci., vol. 1, pp. 339-358, 1994. 
[22] G. N. Patel, E. A. Brown, and S. P. DeWeerth, "A Neuromorphic VLSI System for Modeling the Neural Control of Axial Locomotion," in Advances in Neural Information Processing. MIT Press, Cambridge, MA, 1999, vol. 11.

[23] M. A. Lewis, R. Etienne-Cummings, A. H. Cohen, and M. Hartmann, "Toward Biomorphic Control Using Custom aVLSI CPG Chips," in International Conference on Robotics and Automation, San Francisco, CA, 2000.

[24] M. A. Lewis, R. Etienne-Cummings, M. Hartmann, Z. R. Xu, and A. H. Cohen, "An in silico central pattern generator: silicon oscillator, coupling, entrainment, and physical computation," Biological Cybernetics, vol. 88, pp. 137-151, 2003.

[25] F. Tenore, R. Etienne-Cummings, and M. A. Lewis, "Entrainment of silicon central pattern generators for legged locomotory control," in Advances in Neural Information Processing Systems 16, S. Thrun, L. Saul, and B. Schölkopf, Eds. Cambridge, MA: MIT Press, 2004.

[26] K. Nakada, T. Asai, and Y. Amemiya, "An Analog CMOS Central Pattern Generator for Interlimb Coordination in Quadruped Locomotion,” IEEE Transactions on Neural Networks, vol. 14, no. 5, p. 1356, 2003.

[27] B. Hasslacher and M. W. Tilden, "Living Machines," in Robotics and Autonomous Systems: The Biology and Technology of Intelligent Autonomous Agents, L. Steels, Ed. Elsevier, 1995.

[28] S. Still, "Walking gait control for four-legged robots," 2000, phD Thesis, Swiss Federal Institute of Technology, Department of Physics.

[29] P. Gambaryan, How Mammals Run: Anatomical Adaptations. Wiley, New York, 1974.

[30] N. H. E. Weste and K. Eshraghian, Principles of CMOS VLSI Design. Addison Wesley, 1993.

[31] C. Mead, Analog VLSI and Neural Systems. Reading, MA: Addison-Wesley, 1989.

[32] M. Golubitsky, I. Stewart, P. L. Buono, and J. J. Collins, "Symmetry in locomotor central pattern generators and animal gaits," Nature, vol. 401, pp. 639-635, 1999.

[33] G. Andreou, K. Boahen, P. O. Pouliquen, A. Pavasović, R. E. Jenkins, and K. Strohbehn, "Current-Mode Subthreshold MOS Circuits for Analog VLSI Neural Systems," IEEE Transactions on Neural Networks, vol. 2, no. 2, pp. 205-213, 1991.

[34] B. Sch?lkopf, J. Platt, J. Shawe-Taylor, A. Smola, and R. Williamson, "Estimating the support of a high-dimensional distribution.” Neural Computation, vol. 13, no. 7, pp. 1443-1471, 2001.

[35] C. Rasche and R. J. Douglas, "Forward- and Backpropagation in a Silicon Dendrite," IEEE Transactions on Neural Networks, vol. 12, no. 2, 2001.

[36] S.-C. Liu and R. J. Douglas, "Temporal Coding in a Silicon Network of Integrate-and-Fire Neurons," IEEE Transactions on Neural Networks, vol. 15, no. 5, 2004. 